\title{
Norois
}

Environnement, aménagement, société

198 | 2006/1

Géosymbole, écologie, renouvellement urbain, modélisation

\section{Qualité urbaine et ville durable à l'épreuve du renouvellement urbain. L'exemple du Grand Projet de Ville Malakoff Pré Gauchet à Nantes}

Urban quality and sustainable city in the urban renewal. The case ot the urban project Malakoff Pré Gauchet in Nantes (France)

\section{Marc Dumont et Dominique Andrieu}

\section{OpenEdition}

Journals

Édition électronique

URL : http://journals.openedition.org/norois/2004

DOI : $10.4000 /$ norois. 2004

ISBN : 978-2-7535-1546-8

ISSN : $1760-8546$

Éditeur

Presses universitaires de Rennes

Édition imprimée

Date de publication : 1 mars 2006

Pagination : 7-19

ISBN : 978-2-7535-0307-6

ISSN : 0029-182X

Référence électronique

Marc Dumont et Dominique Andrieu, «Qualité urbaine et ville durable à l'épreuve du renouvellement

urbain. L'exemple du Grand Projet de Ville Malakoff Pré Gauchet à Nantes », Norois [En ligne],

198 | 2006/1, mis en ligne le 13 décembre 2008, consulté le 19 avril 2019. URL : http://

journals.openedition.org/norois/2004 ; DOI : 10.4000/norois.2004 


\title{
QuALiTÉ URBAine ET VILLE DURABLE
}

\section{À L'ÉPREUVE DU RENOUVELLEMENT URBAIN.}

\section{L’exemple du Grand Projet de Ville Malakoff Pré Gauchet à Nantes}

\author{
Marc Dumont
}

Laboratoire Actions Urbaines Usages Altérité - École nationale supérieure d’architeCture de Nantes Laboratolre ChôRos - ÉCOLE POLYTECHNique FÉdérale de LAUSANNE (EPFL)

marc.dumont@epfl.ch

DOMINIQUe ANDRIEU

Maison des sciences de L’Homme et de la Société (Tours)

\begin{abstract}
RÉSUMÉ
Les nouvelles politiques urbaines engagées par les métropoles françaises manient abondamment nombre de thématiques en vogue, comme celles du renouvellement urbain et $d u$ développement durable, lequel reste encore surtout utilisé sur mode de l'incantation et du faire-valoir de pratiques urbanistiques. Toutefois, au-delà de leurs aspects à premier abord consensuels, ces thématiques peuvent surgir en confrontation à l'occasion de grandes opérations urbaines et révéler par-là même les enjeux, mécanismes, contraintes et logiques spatiales de la production de la ville contemporaine. C'est le cas du Grand Projet de Ville (GPV) Malakoff Pré Gauchet, une opération visant à transformer un quartier dit sensible et actuellement en phase opérationnelle dans la métropole nantaise, dans laquelle une confrontation de cet ordre se révèle à l'occasion du traitement de la qualité architecturale des produits immobiliers censés contribuer au renouvellement de l'offre et de la qualité urbaine d'un secteur de ville. Les démarches sont-elles pour autant radicalement antagoniques? La recherche d'une qualité urbaine tant architecturale qu'environnementale restet-elle une démarche à haute valeur ajoutée incompatible avec la question du traitement des espaces publics de proximité, la transformation des grands ensembles se situe-t-elle irrémédiablement dans d'autres sphères que celles de la sortie de nouveaux programmes immobiliers? C'est sur ces différentes questions que le présent article souhaite apporter une série d'éclairages en s'appuyant sur une approche ethnographique d'un projet urbain qui couple une lecture des transformations concrètes avec des observations de réunions et de discussions entre maîtrises d'ouvrage, maîtrises d'ouvres et concessionnaires. Il s'agit ici moins tant de viser à y formuler des solutions substantielles que de souligner les différents défis qui, par l'espace, se posent désormais à l'aménagement de quartiers urbains.
\end{abstract}

Mots CLÉS : Développement durable-Métropoles - Nantes - Pratiques urbanistiques - Projet social - Renouvellement urbain - Ville durable. 


\section{ABSTRACT \\ Urban quality and sustainable city in the urban renewal. The case ot the urban project Malakoff Pré Gauchet in Nantes (France)}

The new urban development policies of the French metropolises broadly invested a various number of themes, particularly that of sustainable development which, while still employed more as a marketing argument of town planning practices, is nevertheless present in large scale urban operations.

Sometimes, sustainable development joins the problems of urban renewal, as part of operations aiming at the transformation of so-called shaded quarters. It is the case of operation Malakoff Pré Gauchet, at present in operational stage in Nantes city, notably in the context of the treatment of architectural quality through the realization of property products, which should contribute to the renewal of the urban offer of this city area. But are these approaches indeed radically antagonistic? Do social projects and "sustainable City" remain practices with high added value incompatible with the question of the management of public areas, with that of the transformation of large housing estates or with that of new property programs?

By following a pragmatic approach, which favors observations of meetings and debates between diverse operators of development, this article underlines how agreements can take shape beyond the difference of cultures (technical, juridical) and identifies the spatial challenges that arise today in the context of the development of urban quarters.

KEY WORDS : Metropolis - Nantes (city) - Urban Planning - Social Project - Sustainable City - Sustainable Development - Urban Renewal.

Le renouvellement urbain et le développement durable font partie des thématiques investies par les métropoles dans le cadre de leurs pratiques urbanistiques (Dumont, 2004). Ils sont une entrée particulièrement pertinente pour interroger les relations qui s'établissent entre deux grandes logiques actuelles assez différentes de développement urbain que l'on retrouve pourtant parfois simultanément à l'œuvre à l'intérieur de situations de projet. Ces logiques de développement sont en premier lieu celles des projets sociaux et du développement social plus en général : elles concernent en France les pratiques d'aménagement qui visent à transformer les conditions de vie de populations dans le cadre de la Politique de la Ville, populations qui, plus que d'autres, se retrouvent aux prises avec des difficultés matérielles, environnementales et humaines (intégration, marginalisation). Ce sont, par ailleurs, les logiques propres aux pratiques de développement socioéconomique, dont l'objectif est de produire une offre urbaine de qualité par des actions en direction de l'environnement physique, d'amélioration du cadre bâti et de son contexte urbain. Pour quelles raisons et de quelle manière peut-on donc examiner ces relations, quel en est l'intérêt?

La première raison est assez simple : il y a en effet a priori une incompatibilité radicale entre l'amélioration de la qualité de vie et de l'environnement souvent considérée comme l'apanage des classes aisées dans les pays dits développés pour les coûts importants qu'elle implique, l'intérêt qu'elle suscite, et de l'autre les aspirations de classes sociales précaires qui n'auraient que faire d'entendre parler de «développement durable». Le premier enjeu consiste donc à réexaminer cette bipartition pour vérifier si elle ne se pose pas en des termes autrement plus complexes dans le cadre quotidien de l'aménagement opérationnel. Ce cadre restant par ailleurs souvent méconnu par les analyses géographiques ${ }^{1}$, la seconde raison est très clairement celle d'un enjeu de connais-

1. Ce point n'est pas surprenant. C'est moins le caractère souvent superficiel de la connaissance des procédures effectives d'aménagement telle qu'elle est dispensée par la géographie qui est dommage, que la dilution d'un travail d'élucidation de logiques spatiales qui lui est spécifique et dont elle devrait tirer pourtant toute son entière légitimité. Il est regrettable que 
sance. Puis, plus fondamentalement, la justification d'une telle entreprise de connaissance réside dans un pari spatial, c'est-à-dire dans l'hypothèse que des situations de projet sont à même de donner à penser à la fois sur des logiques propres à l'aménagement urbain contemporain mais aussi plus largement sur la géographie d'une métropole. L'intérêt de l'éclairage spatial d'une analyse des relations entre différentes logiques de développement à l'occasion d'opérations urbaines réside à partir de là dans un lien étroit posé entre le local et le global. Il consiste à affirmer que des situations locales voire hyper-localisées sont à même d'éclairer sur des logiques plus profondes qui se jouent à des échelles plus vastes que celles du cadre de simples réunions, par exemple, une approche par des situations de projet dont l'efficacité a déjà été montrée (Dumont, 2005).

La perspective vise en ce sens à être à la fois scientifique en vue d'identifier ce que le local apprend sur le global mais aussi orientée vers l'action, pour saisir comment le local interpelle sur des questions plus globales.

Dans cet objectif, nous partirons de situations d'aménagement à l'intérieur desquelles se posent les questions du renouvellement urbain et du développement durable via le thème de la ville durable, en prenant pour objet d'étude le contexte offert par le Grand Projet de Ville Malakoff Pré Gauchet à Nantes. Cette réflexion s'appuie sur une expérience réalisée dans le cadre d'un collectif de recherche ${ }^{2}$ : une observation d'un «projet urbain en train de se faire » (ethnographie de l'urbanisme) consistant à suivre au plus près un projet dans son élaboration et sa concrétisation, des réunions les plus techniques au plus officielles, de validation comme de décision tout en assurant une documentation des transformations effectives de l'espace urbain.

Il s'agira de présenter cette opération de développement urbain dans laquelle se nouent les problématiques de développement durable et social, puis de suivre à travers deux situations de production d'une qualité urbaine, le jeu et la circulation du modèle de la «ville durable ». Enfin, nous soulignerons à la fois les logiques que cette circulation révèle, mais aussi, dans une perspective plus centrée sur l'action, nous tenterons de dégager les différentes logiques et enjeux spatiaux qu'elles permettent de pointer.

\section{Entre développement durable et social : le GPV Malakoff Pré Gauchet}

A Nantes, le projet urbain Malakoff Pré Gauchet concerne une vaste opération de Renouvellement Urbain réalisée dans le cadre de la Politique de la Ville subdivisée en deux axes d'action : la transformation d'un secteur d'habitat existant (démolition - réhabilitation - reconstruction) et la valorisation d'une zone de friche industrielle dans des objectifs de production d'un nouveau quartier urbain d'habitat et d'immobilier tertiaire, EuroNantes. Cette bipartition se traduit dans l'organisation même des dispositifs institutionnels du projet avec la distinction entre une maîtrise d'ouvrage directe conservée par l'institution - la Communauté Urbaine Nantes Métropole - sur un quartier existant (Malakoff), et l'aménagement du second secteur délégué à un aménageur dans le cadre juridique d'une Zone d'Aménagement Concertée (Pré Gauchet) ${ }^{3}$.

\section{UNE INDISPENSABLE CLARIFICATION SÉMANTIQUE}

Il importe avant tout de préciser les utilisations des deux expressions de développement durable et de renouvellement urbain réalisées dans le cadre de cet article. Sans surprise, le flottement

cette activité d'élucidation s'efface trop souvent au profit d'une méta-science assez vague des «systèmes d'acteurs » qui inclurait rien moins l'urbanisme, les sciences politiques, l'aménagement, l'architecture et bien d'autres.

2. Ce programme de recherche d'analyse très concrète de «la ville en train de se faire », dirigé par Laurent Devisme, s’inclut dans un dispositif plus large d'une Plate-forme d'Observation des Projets et Stratégies Urbaines à l'échelle européenne (POPSU), lancé par le ministère de l'Équipement (PUCA). L'équipe, pluridiciplinaire (architecture, sociologie, sciencepolitique, aménagement, géographie) du Laboratoire Architecture Usage Altérité de l'école d'architecture de Nantes mène actuellement une analyse comparative de deux grands projets urbains sur l'agglomération de Nantes, le projet cité et le Projet de l'Île de Nantes.

3. Sur le système de la ZAC et de ces enjeux, cf. S. Demeure et al., 2004. 
sémantique important de l'expression « développement durable » est noté par un nombre important de travaux. Certains auteurs avancent (De Biasi, 2006) que c'est de ce flottement sur sa signification exacte que le terme tirerait son efficacité : il peut être utilisé comme faire-valoir systématique tant des pratiques politiques, sociales, environnementales ou économiques, que du champ scientifique dont il se transforme en gage de sérieux et d'actualité des recherches ${ }^{4}$. Plus donc qu'à proposer une nouvelle définition de la notion, nous en proposerons une approche pragmatique qui vise à élucider ses définitions à partir des usages qu'en font des acteurs de projets urbains. Il ne s'agit pas d'une dérobade mais d'une position théorique et méthodologique. Théorique tout d'abord : cette perspective pragmatique s'inscrit dans les travaux de la sociologie de la traduction (Callon et al., 2001) pour lesquels les opérations de qualification que font les acteurs sociaux en ont aussi beaucoup à nous apprendre sur des réalités considérées, parfois au moins autant que les définitions savantes ou plus objectives que les chercheurs peuvent produire. Pour circonscrire une définition, on s'attache dès lors à la manière dont ce terme circule, est utilisé, circule dans les mondes de l'action, sert à qualifier ou caractériser des états, des stratégies etc. et s'inscrit plus largement dans une activité de production de l'urbain. La position est aussi méthodologique puisqu'elle invite à suivre au plus près les acteurs, elle rejoint une approche par les « situations » qui va être présentée.

Inversement, du côté du renouvellement urbain, le flottement doit impérativement être levé à cause de brouillages récents intervenus dans une notion stabilisée. La problématique du renouvellement urbain est loin d'être nouvelle : dès les années 1970, nombre de travaux avaient éclairés sur des pratiques de transformation de la ville sur elle-même qui réinvestissaient des secteurs urbains en déprise pour des raisons d'opportunité de développement culturel (Webman, 1982) ou de dégradation importantes des conditions résidentielles, de transport et d'équipement (Gibson et Langstaff, 1982) 5 .

Au cours des années 2000, le terme fait irruption dans la Politique de la Ville en France, cet ensemble de politiques publiques lancées à partir de 1981 en direction des quartiers urbains traversés par des difficultés tant sociales, résidentielles qu'économiques. Lancé à l'occasion de la loi Solidarité Renouvellement Urbain (2000) puis prolongé par la Rénovation Urbain sous le ministère de Jean-Louis Borloo, le Renouvellement Urbain (également avec des majuscules) marque un tournant radical dans ces politiques (Anderson et Vieillard-Baron, 2000) : après une séquence centrée sur l'amélioration du cadre de quartier, puis une autre de réhabilitation, l'expression désigne alors une phase de démolitions/reconstructions, souvent spectaculaires (Baudin et Genestier, 2002) et difficilement vécues par les résidents des grands ensembles concernés.

\section{LES DEUX VOLETS D’UNE OPÉRATION COMPLEXE DE DÉVELOPPEMENT URBAIN}

Le quartier Malakoff Pré Gauchet est une « cité extrêmement proche du centre décisionnel » (Garat et al., 2005) et de l'hypercentre de Nantes; il est le pur produit d'une logique de sectorisation : découpé au sud par un grand boulevard urbain et à ses autres bords géographiques par trois lignes de chemin de fer (fig. 1), il contribue à la configuration nantaise d'une « ville duale ${ }^{6}$ », d'une dynamique urbaine à double jeu (Rapetti, 2004). Si l'on y note la présence incontestable de contraintes physiques (proximité de terrains inondables) et d'une concentration de situations sociales précaires (taux

4. Le nombre de publications sur ce sujet est impressionnant. On recommandera toutefois en particulier deux récents ouvrages collectifs qui lui sont consacrés et contribuent à clarifier les différents problèmes qui se posent à travers ce terme. Cf. P. Knoepfel et al., 2005, ainsi que M.-C. Smouts et al., 2005.

5. On reverra plus particulièrement au chapitre introductif de l'ouvrage qui montre l'étonnante persistance trait pour trait des mêmes questions près de tente ans plus tard. Plus récemment et en complément de ce terme, le thème de la régénération urbaine est revenu en force dans les politiques urbaines pour désigner des pratiques urbanistiques investissant plus spécifiquement des friches urbaines. Sur cette question de la régénération urbaine, signalons en particulier une thèse de doctorat qui lui est actuellement consacrée par la géographe Aude Chasseriau (La régénération urbaine. Diffusion et évolution d'un concept. Étude comparée en France et en Grande-Bretagne).

6. Ibidem, p. 134. 
de chômage élevé), elles ne constituent pas pour autant un « défaut congénital » (Luneau, 2003) : le territoire dispose d'atouts dont une intense vie de quartier, la proximité évoquée du centre-ville et la présence d'un espace naturel classé Natura 2000 (la Petite Amazonie). Son périmètre, classé Zone Urbaine Sensible (ZUS), a connu l'ensemble des procédures propres à la Politique de la Ville : intégré au Contrat de ville puis Grand Projet de Ville en $2000^{7}$, il est actuellement l'objet d'une convention de Renouvellement Urbain signée en décembre 2004 avec l'Agence Nationale de Renouvellement Urbain (ANRU), engageant l'Etat, la Région Pays de Loire, le département Loire-Atlatique, les bailleurs sociaux, la Communauté Urbaine et la Ville de Nantes dans un vaste programme d'action. Aux yeux de ces intervenants institutionnels, l'intérêt de la procédure de Grand Projet de Ville serait de permettre le désenclavement du quartier, de produire une diversité des espaces habités, une nouvelle qualité urbaine, et de susciter une dynamique sociale ${ }^{8}$. Dans ce cadre, une série de "volets » sont définis et feront l'objet d'autant de conventions : l'habitat (charte de relogement), le projet de territoire, la démocratie de proximité et le développement de l'accès aux ressources numériques. Deux grands secteurs géographiques sont concernés par cette procédure, Euronantes et Malakoff, chacun ayant des orientations différentes.

Sur le secteur Malakoff proprement dit (divisée en tranches opérationnelles amont, aval et centre), exclusivement composé d'habitat social (98\%, détenus par le même bailleur), quatre grands axes d'action sont définis. Une transformation de l'habitat existant, tout d'abord, donnant lieu à des opérations de démolition/reconstruction (294 logements concernés) ou de réhabilitation des immeubles existant (457 logements), de transformation de leurs abords (résidentialisation). À l'échéance du projet, le quartier comprendra 1600 logements (1300 dans le cahier des charges initial) dont $80 \%$ de logements sociaux contre 1450 actuellement : une diversification de l'habitat est recherchée par la création de surfaces destinées tant au tertiaire quà la réalisation de logement à accession libre. A cela s'ajoute un lourd programme de transformation de l'environnement urbain avec la réalisation d'équipements publics (centre socio-culturel, piscine, construction d'un nouveau collège - gymnase) et d'équipement structurels (franchissements des lignes ferroviaires, création d'un nouveau pont sur la Loire, etc.). En ce sens, le volet affiche le souhait résolu de « conférer une nouvelle qualité urbaine à l'environnement du quartier » en poursuivant des objectifs de développement durable, de rééquilibrer l'offre de logement (surconcentration actuelle de grands logements), de renouveler également fortement les populations résidentes (reloger certaines familles dans d'autres secteurs de la métropole, attirer de nouveaux habitants sur le quartier). Enfin, dernier axe, le projet social de territoire incluant une série de dispositifs de prévention, d'animations, d'action en matière d'emploi et d'insertion.

Du côté d'Euronantes (35 ha), le nouveau quartier envisagé inclut le Vieux Malakoff avec 350 logements prévus à terme (309 logements actuels dont 30 logements sociaux) ainsi qu'une friche industrielle de 15 ha (Pré Gauchet). Cette partie du quartier devrait accueillir à terme un programme de 1300 logements neufs (dont $30 \%$ de logements sociaux - 250 au total) ainsi qu'un programme de $130000 \mathrm{~m}^{2}$ d'immobilier tertiaire (bureaux) attendant l'arrivée de 8000 futurs employés. Cette ligne tertiaire prend place dans le cadre d'un vaste programme d'ensemble d'un total de $400000 \mathrm{~m}^{2}$. La réalisation du nouveau quartier Euronantes Gare constitue en effet une des trois têtes d'un futur quartier d'affaire de la métropole, le «triangle tertiaire », composé de deux autres pôles (ZAC Madeleine-Champs-de-Mars et ancien site du Tripode) dont l'institution souhaite qu'il soit à l'origine d'une nouvelle centralité métropolitaine (fig. 1). La maîtrise d'ouvrage de l'aménagement et de la commercialisation de cette ZAC, tout en étant inclue dans le GPV, est déléguée à Nantes Aménagement, une société d'Économie Mixte (SEM). Le programme affiche d'emblée le souci de produire une qualité architecturale et urbaine exemplaire, les institutions insistant sur leur souhait de l'installer davantage à grande proximité du centre-ville, misant sur les atout de la gare SNCF et du Palais des Congrès (Madeleine Champs de Mars).

7. Sur la différence entre ces dispositifs, cf. A. Anderson et H. Vieillard-Baron, 2000

8. Extrait de la Convention de Renouvellement Urbain, 20 décembre 2004. 


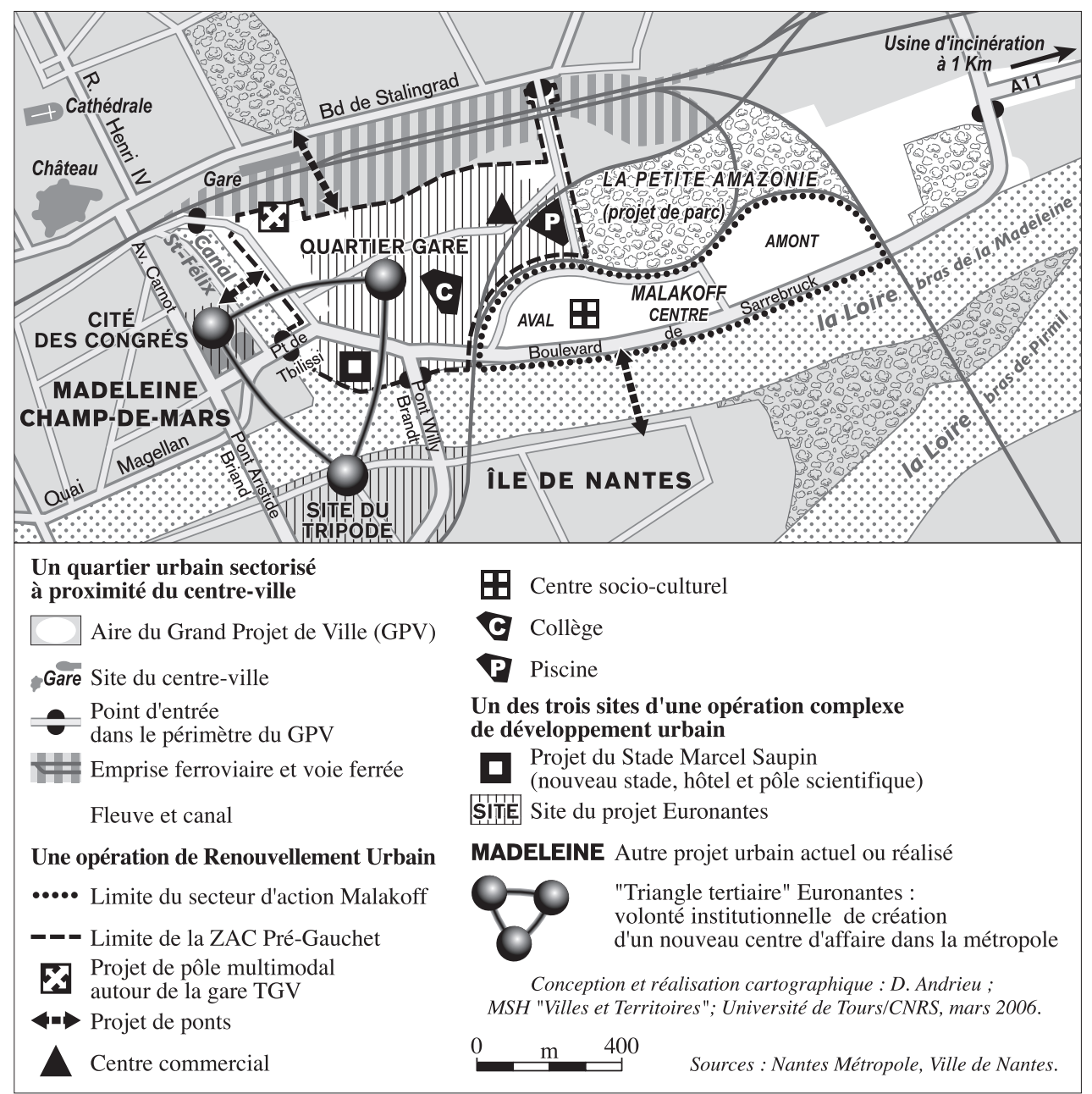

Figure 1 : Le Grand Projet de Ville (GPV) Malakoff Pré-Gauchet : du quartier d'habitat social au quartier d'affaire européen ness District.

The GPV Malakoff Pré-Gauchet : from the social housing district to the Metropolitan Busi-

\section{Qualité urbaine et Ville Durable : deux épreuves de concrétisation de modèles urbanistiques}

Dans ce contexte effervescent qui s'étend des études urbaines préliminaires précédant les conventions (2001-2003) aux phases les plus opérationnelles telles que celles des chantiers de démolitions, de la production des réseaux, des réfections de voiries et des espaces publics à la construction de la nouvelle piscine (2004-2006), les « situations d'habitat » occupent une place tout à fait singulières. Il s'agit de moments qui se cristallisent autour des questions ayant trait aux différentes programmations immobilières et à leurs transformations périphériques (reconfiguration du foncier, notamment dans le cadre de la résidentialisation), au cours desquels il est possible d'observer au plus près le travail des logiques économiques, foncières, de développement durable, financières et sociales. Lorsqu'il s'agit de programmer les phases de démolition, de lancer la réalisation d'un ouvrage d'art 
sur les lignes ferroviaires, de coordonner la mise en place des réseaux (assainissement, électricité, etc.), de concrétiser un Pôle Multimodal d'Echange ${ }^{9}$, comme dans tout projet urbain d'envergure, on voit apparaître dans les réunions organisées par les maîtrises d'ouvrages et celles qui leurs sont périphériques, un grand nombre d'acteurs techniques, des experts, des promoteurs immobiliers, des cabinets de consultants, amenés à travailler ensemble. Le suivi de l'ensemble de ces réunions tant de préparation des orientations que des phases opérationnelles (coordination de maîtrise d'ouvrage, maîtrise d'œuvre, chantiers) constituait le travail quotidien de cette recherche. C'est à l'intérieur des débats qui ont lieu à cette occasion entre acteurs et partenaires et qui accompagnent parfois « en temps direct » les transformations concrètes liées au projet, que la circulation de normes de qualité urbaine telle que la Haute Qualité Environnementale (HQE) ou liées au modèle de la «ville durable » a pu être observée, ainsi qu'au cours des réunions de mise en scène des projets (présentation du quartier à la rencontre internationale des promoteurs immobiliers, le MIPIM, par exemple). Quel type de produit immobilier sortir? Comment assurer la rentabilité de l'opération? Faut-il où non respecter les attentes institutionnelle d'un taux de logement social élevé sur certaines parties du quartier? Tous ces débat sont récurrents dans les «situations d'habitats » : ils mettent en lumière le jeu antagonique ou complémentaire des deux logiques de développement, et sont éclairants sur les logiques spatiales de la production contemporaine de l'urbain ${ }^{10}$.

\section{DE LA HQE AU LABEL « QUALITEL » : QUALITÉ OU MIXITÉ URBAINE?}

Premier ensemble d'observation, la réalisation d'un cahier des charges constituant l'ensemble proprement dit de l'appel aux promoteurs-concepteurs immobiliers pour la commercialisation de la ZAC intégrée au GPV, orchestré par la SEM Nantes Aménagement. Ce cahier, classique en aménagement, se compose de documents variables selon les opérations et les nouvelles législations; en l'occurrence, il rassemble un cahier des prescriptions de chantier, un cahier des charges de cession des terrains, et surtout d'un cahier des charges des prescriptions architecturales et urbaines des îlots constructibles cadrant très précisément les attendus de l'offre urbaine pour tout promoteur intéressé par l'opération. La réalisation de l'appel aux promoteurs-concepteurs a donc consisté à établir ces documents présentant l'opération, ses orientations architecturales et urbaines, les attendus de la Collectivité, les balises juridiques et normatives, puis l'appel en tant que tel a eu lieu, suivi des jurys sur les propositions rendues.

Cette séquence a été très instructive sur une mécanique de gouvernance : la méthode de travail initiée par le chargé d'opération n'a pas pris la forme d'une simple duplication adaptée de cahiers préexistant ni à produire exclusivement à l'interne, dans le seul cadre de la SEM, son contenu. Elle consistait à mettre ensemble autour d'une table des cultures du projet très différentes: architectes, chargés de projets, chargé de mission environnement, bailleurs sociaux, promoteurs immobiliers, notaire, avocat, responsables de la mission logement etc. Elle visait aussi, sur un plan plus opérationnel, et tout en fonctionnant très à tâtons, à produire une véritable réflexion autour de pratiques de simulation du foncier, à explorer de montages juridiques et financiers innovants, donc à constituer une sorte de « laboratoire de la fabrique urbaine ». Le suivi de son élaboration nous a mené dans les corridors et lieux de production du système du logement, que ce soit ceux du Club Immobilier Nantes Atlantique (CINA) ${ }^{11}$, d'une Commission Locale du Logement ${ }^{12}$, d'experts mobilisées sur le relogement, et naturellement, ceux des institutions (Communauté Urbaine, Ville de Nantes) et opérateurs d'aménagement (SEM).

9. Celui-ci se situe autour de la gare SCNF et vise à réagencer l'ensemble des déplacements (voitures, transports en commun, taxis) en vue notamment de desservir efficacement le nouveau quartier.

10. Marché International des Professionnels de l'Immobilier, qui se tient en France à Cannes chaque année.

11. Celui-ci rassemble les principaux promoteurs-concepteurs locaux. Créé en 1992, il se définit lui-même une mission d'information et de réflexion aux problèmes touchant à l'Immobilier et à l'Urbanisme dans la métropole.

12. Ces commissions ont été instituées dans le cadre du Grand Projet de Ville, par la Ville de Nantes, pour assurer la coordination de l'opération de relogement entre les bailleurs sociaux, la Ville de Nantes, et les associations de locataires. 
La question de la qualité urbaine va alors se cristalliser autour d'un débat sur la norme HQE ${ }^{13}$ qui y fait son apparition avec l'invitation à l'une des réunions des représentants de la Mission «Environnement» de la Communauté Urbaine ${ }^{14}$. Ceux-ci annoncent le souhait résolu de deux élus que la norme soit présente dans les programmes immobiliers (îlots) sortis. Elle émergera également comme norme de qualification imposée aux chantiers de construction en se surimposant à la directive thermique RT $2005^{15}$. Tous les échanges qui ont lieu entre les différents partenaires au cours de réunions de coordination de maîtrise d'ouvrage ou encore rassemblant les directions générales des Projets Urbains visent à partir de là à s'ajuster aux surcoûts liés à ces exigences, mais surtout à construire une réponse à une tension qui va apparaître entre une ligne sociale et une autre plus qualitative du projet.

En effet, d'un côté se situent les objectifs politiques affichés clairement par le Président de la Communauté Urbaine, Jean-Marc Ayrault, et par tous les signataires de la convention ANRU souhaitant intégrer dans l'ensemble du projet Euronantes un fort quotient de logement social (entre 30 et $35 \%$ ), un chiffre dépassant nettement le quota imposé par la loi Solidarité Renouvellement Urbain $(\mathrm{SRU})^{16}$. De la part des acteurs politiques ce chiffre marque une volonté forte de contrecarrer les possibles dérives d'une opération située à une telle proximité du centre-ville, et de donner une assise concrète à un impératif de mixité souvent affiché de manière incantatoire, en évitant la dualité entre un «pôle d'affaire » et le secteur Malakoff.

Ce souci de mixité par la diversification de l'habitat heurte une autre logique, économique, de quartier d'affaire, qui s'orientait vers une valorisation financière maximale du site. La certification HQE (s'ajoutant à des contraintes comme la RT 2000-2005) ${ }^{17}$ devient l'élément central autour duquel se cristallise une forte tension. Celle-ci se concentre d'abord dans un débat sur la dénomination: pour les uns «Pré Gauchet »(désignation juridique de la ZAC) plus urbain et social, pour les autres «Euronantes Gare », un nom choisit par la Mission en charge du Développement économique censé doter le secteur d'une vocation prestigieuse au moins européenne ${ }^{18}$. Puis, elle est à l'origine d'un rapport de force assez dur entre les bailleurs sociaux et les promoteurs immobiliers que la SEM doit amener à s'entendre. Le programme prévisionnel de logement dans sa première phase vise un total de 512 logements dont 162 de locatif social, 185 de locatif libre, 41 en accession sociale et 124 en accession libre, différenciant trois types d'accession : l'accession sociale réglementée (primo-accédant) ${ }^{19}$, le milieu de gamme et le haut de gamme, sur des logements qui s'étendent du T1 au T5+. Ce n'est pas tant le coût d'acquisition des terrains ${ }^{20}$ que les prix de sortie imposés par la collectivité qui posent problème au niveau des réponses attendues, en termes de prix-valeurs de sortie, de loyers envisagés par type de produits, et des prix de sortie en VEFA $^{21}$ pour le logement social.

13. Cette norme impose un certain nombre de contraintes à la réalisation des bâtiments, afin que leur production préserve l'environnement.

14. Sur le plan administratif, la Communauté urbaine est divisée en Missions, équivalent des « services » qui se répartissent l'urbanisme, les transports, l'environnement etc.

15. La norme thermique RT 2005 impose un certain nombre de contraintes aux bâtiments en vue de contribuer à la réduction des pertes d'énergie (chaleur), principalement par les façades. Du côté des chantiers, ceux-ci doivent respecter un certain nombre de normes, en particulier, et c'est en l'occurrence le cas, des normes qui en font des « chantier HQE » qui visent à limiter les risques et les nuisances pour les riverains du chantier, la santé des ouvriers, les pollutions liées au chantier etc., normes définies dans les cahiers des charges de la ZAC.

16. Cette loi imposait en effet aux agglomérations de plus de 50000 habitants, de construire au moins $20 \%$ de logements sociaux, pour répondre aux impératifs de «mixité ».

17. La RT 2000 était moins exigeante que la RT 2005, cf. supra.

18. Trait pour trait, on retrouvera cette divergence d'ambition à l'occasion la construction d'un nouveau centre commercial

19. Les primo-accédant, personnes ou couple n'ayant jamais été propriétaires de leur vie, disposent en effet d'aides financières conséquentes pour réaliser leur première accession à la propriété.

20. En moyenne, les charges foncières attendues par la SEM sont de 180 euros HT/m² de Surface Hors Euvre Nette (SHON) pour le locatif social, 220 euros $\mathrm{HT} / \mathrm{m}^{2}$ de SHON pour le locatif intermédiaire et l'accession sociale, 260 euros HT/m² de SHON pour l'accession libre.

21. Vente en l'Etat de Futur Achèvement. 
D’un côté les opérateurs HLM (bailleurs sociaux) s’insurgent en soulevant leur incapacité à s’investir sur des logements dont ils jugent les coûts de sortie trop élevés, de l'autre, les promoteurs immobiliers s'inquiètent de l'impossible viabilité d'une opération dont on leur impose des prix de sortie qu'ils jugent très inférieurs à ceux du marché. L'imposition d'un prix de sortie très bas pour le logement social (inférieur à 1000 euros le $\mathrm{m}^{2}$ ) - condition première pour l'accession sociale qu'elle soit ou non de la primo-accédant -, renverrait dès lors pour eux à des exigences de péréquations accentuant le différentiel de l'offre (récupérer sur les logements libres ce qu'ils considère comme les ventes à perte des logements sociaux). Une telle situation percute les objectifs politiques qui étaient de "faire revenir les classes moyennes en centre-ville » et d'éviter la reproduction interne au quartier d'un décrochage entre des catégories sociales aisées et d'autres nettement moins. Il s'agit là d'un « cercle vicieux » puisque les promoteurs se tournent alors vers la Collectivité en lui demandant d'assumer le différentiel de coût, ce que ses finances locales ne lui permettent pas.

Face à ce risque et après plusieurs mois de réflexions, une solution intermédiaire sera retenue par les élus : non pas la norme HQE mais l'exigence de certification Qualitel, intégrée dans l'appel aux promoteurs, moins exigeante, promoteur et bailleurs finissant par s'entendre sur un coût moyen de 1300 euros le $\mathrm{m}^{2}$ de logement social. Ce moment révèle la complexité d'une traduction en termes opérationnels concrets des principes de mixité urbaine, sous-estimée par les critiques adressées à ce principe. Pourtant, dans le cas précis, force est de noter une réussite : l'exploration de complexes montages juridiques et financiers, en descendant à l'échelle du bâtiment, a permis de mixer des programmes de logement, prouvant qu'il est possible de réaliser des offres urbaines non-segmentées ${ }^{22}$.

Dans les actes et confrontée à son concrétisation, la mixité urbaine urbaine ${ }^{23}$ est autrement plus complexe qu'une simple rhétorique d'action publique. Au final c'est moins la question du coût liés aux normes environnementales qui accentue la tension, que la pression foncière qui pèse sur des terrains à grande proximité du centre-ville, dans un contexte de crise du logement.

\section{PARIER SUR LA « VILLE DURABLE » OU SE RETRANCHER SUR LA « VILLE RENTABLE »?}

La seconde situation urbanistique observée est liée au modèle urbanistique de la « ville durable » (Theys et Emelianoff, 2001), que les intervenants du projet tentent étape après étape de produire au cours des séquences de reconstruction sur le secteur Malakoff (amont, centre et aval).

De manière récurrente, les élus rappellent leur souci de corriger les erreurs des années 196070 en France qui auraient consisté à élaborer dans l'urgence les vastes programmes immobiliers à l'origine des grands ensembles, jugés responsables des dysfonctionnements sociaux du secteur. Construire une réflexion sur un quartier qui intègre la question du développement durable consiste donc pour eux à anticiper l'évolution de la ville, en construisant notamment pas à pas un projet urbain, sans décision tranchée, mais de manière itérative. Comment se concrétise ce modèle de « la ville durable » en tant que réfléchie sur le long terme? Sa production peut se suivre à travers trois micro-moments - la réalisation des terrassement et travaux de voirie, les opérations de résidentialisation et la mise en place d'un réseau de chaleur urbain - et, en ce sens, la chute entre les orientations théoriques et la réalité opérationnelle est parfois un peu rude.

Du côté des terrassements et travaux de voirie, la mise en place des réseaux (électricité, gaz, etc.) oblige leurs concessionnaires à se coordonner avec les maîtrises d'œuvre et d'ouvrage, pour réaliser les phasages de chantiers, à en définir aussi les modalités exactes. Lorsqu'il s'agit de mettre en place des réseaux sur des secteurs urbanisables dans le futur et lorsque se négocie notamment la pose de fourreaux protecteurs des câbles, les discussions se concentrent sur la « mutabilité » des espaces tant du

22. Si les offres sont habituellement toujours segmentées, les montages retenus permettent précisément de dépasser cette situation en mixant à l'interne : un appartement en logement social couplé avec un appartement en accession libre, par exemple, dans le même complexe d'habitation, une situation jusque-là impensable dans le cadre d'une tour HLM, par exemple.

23. Que l'on différencie ici très clairement de la mixité sociale avec laquelle elle est souvent confondue. 
côté de la maîtrise d'œuvre dont la maîtrise d'ouvrage attend qu'elle intègre la dimension évolutive du projet, que des concessionnaires qui sont assez peu enclins à poser des réseaux non-immédiatement rentables (eau, électricité, etc.). Il est inconcevable pour les concessionnaires de poser des câbles qui serviront peut-être dans dix ans lors de l'extension de l'urbanisation du secteur, l'agencement du réseau dont son évolution dépend ne pouvant se faire qu’à cette occasion. La question est ici encore financière : qui assume le surcoût immédiat d'une opération rentable qu'à moyen ou long terme? Qui serait prêt à assumer des coûts sans bénéficier des retours sur investissement? La question déstabilise la Communauté Urbaine confrontée à des exigences de visibilité rapide, source de légitimité (Charrié, 1996). Pourquoi, par ailleurs, ce flottement sur l'éventuelle extension du quartier? Parce que se maintient le risque d'un échec des opérations immobilières situées à proximité dont cette évolution dépend. La « mutabilité » se trouve ainsi au cœur d'un pari à la fois financier et stratégique dépendant de conjonctures qui dépassent le seul cadre de la métropole nantaise.

Second moment, celui des opérations dites de résidentialisation qui consistent à reconfigurer l'organisation de la domanialité publique dans les secteurs « Contrat de ville » de l'agglomération, à repréciser les limites séparatives de gestion entre ce qui est du ressort des bailleurs sociaux et de la puissance publique. Dans ce cadre, la réalisation de nouvelles voiries sur des portions dont la Communauté Urbaine dispose désormais de la maîtrise exige un certain nombre d'opérations de terrassement des sols, de préparation des voiries. Un petit moment conflictuel voit s'opposer l'entreprise de Bâtiments et Travaux Publics ayant en charge la réalisation de ces opérations avec son commanditaire public. L'entreprise préfère utiliser les matériaux de remblaiement préparés dans une carrière située à l'autre extrémité de l'agglomération, plutôt que de réutiliser des matériaux issus de la démolition d'une des tours située dans le quartier, à quelques dizaines de mètres du chantier. Il y a deux raisons à cela : de coût et de qualité, la certification des matériaux à moins de $5 \%$ de polluants ne pouvant être produite par l'entreprise de démolition, celle-ci maintenant malgré tout un coût élevé de revente des matériaux. A cette occasion, les discussions permettent de souligner comment la «ville durable » fait irruption dans une situation aussi anodine, invoquée tant par les représentants de la maîtrise d'ouvrage ( « il faut réutiliser ces matériaux, c'est ça aussi, faire du développement durable») que par l'entreprise de BTP («si l'on ne me garantit pas la qualité de ces matériaux, on met en péril le caractère durable de cette voie ») ${ }^{24}$.

Enfin, troisième moment, le réseau de chaleur urbain. Le GPV se trouve à proximité d'une usine d'incinération des déchets - implantée antérieurement aux phases d'urbanisation - dont la capacité énergétique est non-exploitée. Le projet va intégrer cette usine avec la mise en place d'un réseau de chaleur urbain (respectueux de l'environnement) mais qui, là encore, implique des surcoûts sur chacune des phases, dont celles du quartier Euronantes Gare et du prix de sortie des logements (cf. supra). Il s'agit là d'une autre contrainte que les différents intervenants souhaitent mettre en œuvre dans le cadre de l'appel aux promoteurs, celle d'un raccordement obligatoire à ce réseau de chaleur capable de produire du froid durant la période estivale. C'est ici que la symétrie entre développement social et durable est la plus nette, dans la non-rentabilité immédiate de coûts d'investissement ${ }^{25}$. Ce raccordement n'apporte en effet aucune plue value immédiate au programme sinon en tant que démarche soucieuse de l'environnement. Tout comme le souhait résolument affiché de la mixité sociale (cf. supra), la collectivité se retrouve face à l'exigence de se porter garante d'un pari plus éthique et écologique qu'économique.

\section{Programmation urbaine, mobilisation partenariale : les défis en actes du développement urbain}

Que donne à penser en termes géographiques et donc spatiaux, le parcours de ces situations diversifiées qui s'articulent autour des problématiques de développement durable et social dans 
le cadre territorial d'une métropole? Deux aspects au moins peuvent être soulignés : les enjeux en termes de programmation et de coordination des acteurs, ainsi que le lien entre des questions hyperlocalisées et ponctuelles et des enjeux plus globaux.

\section{Au-DELÀ de LA Mise EN DÉBAT, un ENJEU DE PROGRAMMATION URBAine}

Les situations d'habitat sont révélatrices d'enjeux de pilotage en particulier à travers la question du rapport coût/qualité environnementale des opérations, liée à la redéfinition " pragmatique » telle qu'elle intervient dans un projet en train de se faire, définit comme tel par ses acteurs, et qui s'agence autour de deux questions qui viennent d'être évoquées. Elles émergent à travers les niveaux strictement opérationnels : le réseau de chaleur et la démarche HQE. Autour de chacun de ces «problèmes » (November, 2003; Cefaï et Trom, 2001), on a pu noter à chaque fois un flottement important au niveau des décisions, des hésitations prolongées qui se répercutent sur les temporalités du projet, mais aussi un cloisonnement entre des niveaux opérationnels et techniques et ceux de la décision.

Il s'agissait en l'occurrence de valider les orientations concernant le raccordement ou non du programme au réseau de chaleur urbain lié à la déchetterie, la prise en compte ou non des normes HQE dans les programmes résidentiels, mais aussi le fait de privilégier un transport en site propre sur deux voies urbaines (avenue de la gare/allée du Parc). Le choix de limiter la prise en compte du développement durable au niveau du bâti à la norme Qualitel exprime, à travers cette évolution itérative du projet, la difficulté des élus à se saisir d'un dossier et afficher une cohérence sur l'ensemble du processus, à passer de l'invocation à la concrétisation. Elle révèle aussi plus largement un déficit de programmation urbaine sur l'ensemble de la métropole : la question du logement qui se cristallise à travers cette petite opération n'est que le prisme d'une problématique beaucoup plus vaste d'organisation du logement à l'échelle de la métropole, d'un contexte de pression foncière importante, et de difficile maîtrise du développement urbain avec l'émergence d'une nouvelle couronne (étalement urbain).

En ce sens, tant dans les petites situations ayant trait à la « mutabilité » des espaces urbanisés que celles concernant le programme immobilier, apparaît le lien étroit entre des situations très locales et des logiques plus globales, celles de l'échelle métropolitaine ou plus vaste (Jouve et Lefevre, 2004). Ces moments anodins servent de « perturbateurs » manifestant l'absence ou l'inefficacité d'une réflexion globale sur des objectifs clairs de développement urbain. C'est en ce sens que l'on peut considérer que le développement durable conserve un statut incantatoire puisqu'il ne parvient pas à dépasser le seul stade de sa mise en débat.

\section{PASSER DU « RÉFÉRENT COMMUN » À L'ENGAGEMENT PARTENARIAL : UN ENJEU DE GOUVERNEMENT DU PROJET}

Comment un référent commun peut-il devenir un véritable opérateur d'action publique? Comment la «mixité » et la « durabilité » peuvent-elle correspondre à des instruments convaincants de développement urbain? La réponse est déjà fondamentalement organisationnelle. Sur cet aspect, les situations d'habitat révèlent une forte intra-sectorisation des problématiques au sein de la maîtrise d'ouvrage : les missions environnement très pointues quant à leurs dossiers de certification, les promoteurs sur leurs attentes en termes de commercialisation, les bailleurs sociaux sur leur patrimoine, les missions de logement, sur les populations qu'elles doivent reloger. D'où l'intérêt d'une exploration conjointe de tous les montages juridiques et financiers possibles et de l'existence de lieux alternatifs (ceux de la réalisation des cahiers des charges), permettant de dépasser cette structure ordinaire de l'action publique.

Il serait erroné de réduire la tension évoquée à un affrontement caricatural entre une logique de rentabilité et une logique de projet social. En réalité, c'est l'étroitesse des marges de manœuvre qui se profile à travers la question de la viabilité de l'opération. La maîtrise de la qualité envi- 
ronnementale et urbaine par la collectivité est sans doute un très bel objectif. Mais il se heurte d'abord au fait que la ZAC soit déficitaire (coût des études) puis, surtout, sur une situation sur laquelle elle n'a aucune prise : l'inflation du marché immobilier qui touche autant la métropole que les autres villes françaises. La Collectivité est démunie lorsqu'elle se retrouve confrontée à une épreuve de composition, c'est-à-dire à un moment politique au cours duquel elle doit tisser ensemble les fils de logiques contradictoires (groupes sociaux conflictuels, risque de décrochage entre catégories sociales aisées et pauvres) pour produire une décision ou un projet. Derrière les débats sur le type de montage d'opération de logement social se déroule en fait une question qui concerne toute l'évolution de l'offre à l'échelle de la métropole, la politique de logement, les objectifs du Programme Local de l'Habitat et la révision du Schéma de Cohérence Territoriale, autre logique globale que révèle ce moment. Les représentants des commissions habitat présents dans ces réunions soulignent à cet égard comment il apparaît de plus en plus impossible de contrer une évolution vers la formation d'une quatrième couronne métropolitaine, illustrant, via cette opération révélatrice, la difficile voire impossible maîtrise du développement métropolitain.

Les situations illustrent aussi la vision restreinte d'un ensemble d'acteurs « périphériques » pourtant essentiels au projet qui ne parviennent pas toujours à s'inscrire dans une démarche de projet qu'ils considèrent davantage sous l'angle d'un guichet, d'opportunité d'obtention de financements au coup par coup. Au risque de n'être suivi d'aucun effet, la production de partenariat autour de points ciblés du projet est certes une première étape, mais résiduelle au regard de son « endurance » : produire de la continuité d'engagement. Elaborer des formes de gouvernance en mettant ensemble des acteurs diversifier pour stabiliser un référentiel environnemental et social commun est une chose, construire des formes d'engagement consistant à « prendre date » (Joseph, 2004), c'est-à-dire inscrire ce partenariat dans la durée avec des engagements datés et sans dérobade d'imputabilité, en est une autre, autrement plus difficile mais indispensable.

\section{Conclusion}

Nous étions partis en tant que géographes d'une perspective invitant à suivre les normes de qualité urbaine à travers les opérations de qualification des acteurs. À ce terme du propos, il importe de revenir sur la notion même de développement durable, de proposer une grille de synthèse de ces qualifications permettant d'en clarifier les différents sens circulatoires. Dans l'ensemble de ce projet, le développement durable y apparaît de quatre manières. Comme thème d'action, tout d'abord, en tant que référence parfois omniprésente à l'occasion de processus et le plus souvent activée sur un mode incantatoire. Puis, comme norme d'action publique, impliquant une redéfinition de ses modalités organisationnelle (apparition de nouveaux acteurs) et structurelles (financements, coûts des chantiers). Ensuite, comme norme qualitative de production des environnements bâtis (situations HQE en tant que telles). Enfin, comme instrument de production de "la ville durable » (travail sur les réseaux, la domanialité), de son aménagement. Derrière cette variété des rôles que tient une norme de qualité urbaine se joue donc plus largement la question de la compatibilité des objectifs sociaux et qualitatifs. Contrairement à ce qui peut être souvent avancé, cette question ne se pose donc pas (seulement) en termes financiers, mais aussi organisationnels. Elle exige une forte mobilisation intra-sectorielle des acteurs du projet ainsi qu'une exploration sérieuse des combinaisons techniques, juridiques et financières. C'est à ce prix, relativement exigeant, que pourront alors se produire de manière convaincante de nouvelles formes de développement urbain, à la fois durable et social, que bien-être, qualité urbaine et projet sociaux (Dumont, 2006) n'apparaîtront plus comme antagoniques à l'occasion de leur mise en œuvre opérationnelle. 


\section{Bibliographie}

Anderson (A.), Viellard-Baron (H.), 2000. - La politique de la ville, histoire et organisation, Paris, ASH, $192 \mathrm{p}$.

Baudin (G.), Genestier (P.), 2002. - Banlieue à problèmes : la construction d'un problème social et d'un thème d'action publique, Paris, La Documentation Française, 252 p.

Callon (M.), Lascoumes (P.), Barthe (Y.), 2001. - Agir dans un monde incertain. Essai sur la démocratie technique, Paris, Le Seuil, 397 p.

Charrié (J.-P.) (dir.), 1996. - Villes en Projet. Actes du colloque de 1995, Bordeaux, Maison des Sciences de l'Homme d'Aquitaine, $290 \mathrm{p}$.

Cefaï (D.), Trom (D.) (dir.), 2001. - Les formes de l'action collective, Mobilisations dans des arènes publiques, Paris, Éditions de l'EHESS, 409 p.

De Biasi (P.-M.), 2006. - Lexique de l'actuel. Quelques idées reçues de notre temps, vol. 2, Paris, CalmannLévy, $365 \mathrm{p}$.

Demeure (S.), Martin (J.-Y.), Ricard (M.), 2004. - La ZAC. Zone d'aménagement concerté, Paris, Le Moniteur, $304 \mathrm{p}$.

Dumont (M.), 2004. - « L'aménagement d'une plaine inondable en région tourangelle : des directives nationales aux systèmes normatifs locaux, la transformation d'un régime de contraintes environnementales », dans Scarwell (H. S.), Franchome (M.) (dir.), Contraintes environnementales et gouvernance des territoires, La Tour-d'Aigues, Éditions de l'Aube, p. 79-86.

—, 2005. - « Les micro-territoires dans l'aménagement urbain, objets spatiaux et sociaux paradoxaux : le cas du quartier Bourgogne à Orléans », Norois, n 193, p. 55-67.

—, 2006. - «Esthétique, esthésique urbaine. Bien-être et qualité de ville dans les politiques urbaines françaises », dans Fleuret (S.) (dir.), Espaces, Qualité de vie et Bien-être, Angers, Presses de l'Université d'Angers, p. 169-177.

Garat (I.), Pottier (P.), Guineberteau (Th.), Jousseaume (V.), Madoré (F.), 2005. - Nantes. De la belle endormie au nouvel Eden de l'Ouest, Paris, Economica, 180 p.

GibSON (M.), LANGSTAFF (M.), 1982. - An introduction to urban renewal, London, Hutchinson, 1982, 384 p. Joseph (I.), 2004. - Météor. Les métamorphoses du métro, Paris, Economica, 123 p.

Jouve (B.), Lefevre (C.) (dir), 2004. - Horizons métropolitains, Lausanne, Presses Polytechniques et Universitaires Romandes, $289 \mathrm{p}$.

Knoepfel (P.), Da Cunha (A.), Leresche (J.-P.), Nahrath (S.), 2005. - Enjeux du développement urbain durable. Transformations urbaines, gestion des ressources et gowvernance, Lausanne, Presses Polytechniques et Universitaires Romandes, 444 p.

LunEAu (D.), 2003. - Nantes l'avenir d'une ville, La Tour-d'Aigues, Éditions de L'Aube, 135 p.

RAPETTi (D.), 2004. - «Contre vents et marées : «Nantes Atlantique». La nouvelle donne», M@ppemonde, $\mathrm{n}^{\circ} 74$ [http://mappemonde.mgm.fr/num2/articles/art04205.html].

Smouts (M.-C.), Antoine (S.), Beauchamp (A.), Bourg (D.), 2005. - Le développement durable. Les termes du débat, Paris, Dalloz-Sirey, 375 p.

Theys (J.), Emelianoff (C.), 2001. - «Les contradictions de la ville durable ", Le Débat, n 113 , p. 122-135.

November (V.), 2003. - "L'incendie créateur de quartier ou comment le risque dynamise le territoire », Cahiers de géographie du Québec, vol. 47, n 132, p. 367-388.

WeBman (J.), 1982. - Reviving the industrial city: the politics of urban renewal in Lyon and Birmingham, London, New Jersey, Rutgers University Press, 197 p.

Cet article a été reçu le 22 novembre 2005 et définitivement accepté le 21 mars 2006. 
